\title{
Coinfected cases with equine herpesvirus type 1, 4 and Streptococcus equi subsp. zooepidemicus in throughbred horse
}

\author{
Seong-Guk Kim, Gil-Jae Cho ${ }^{1}$, Min-Hee Cho, Young-Hoan Kim, \\ Hong-Young Lee, Jeong-Hye Choi, Jeong-Hwa Kim, Seong-Kyoon Choi* \\ Gyeongbuk Veterinary Service Laboratory, Daegu 702-911, Korea \\ ${ }^{1}$ College of Veterinary Medicine, Kyungpook National University, Daegu 702-701, Korea
}

(Received 1 April 2011, accepted in revised from 16 June 2011)

\section{Abstract}

The Thoroughbred horse was an approximately 4-years-old castrated male with highly emaciation, nasal epistaxis and subsequently died. Gross necropsy revealed epistaxis and hyperemia on the lung, multiple hemorrhage in muscle, and liver was focally attached to the peritoneum with fibrin. According to polymerase chain reaction (PCR), Equine herpes virus type 1 and 4 (EHV type 1, 4) was detected in the lung and trachea. In bacterial culture from kidney, liver, spleen, muscle and blood, Streptococcus equi subsp. zooepidemicus was isolated. Based on the gross lesion and PCR, this horse was diagnosed as EHV type 1, 4 and $S$. zooepidemicus coinfection.

Key words : EHV-1 and 4, Streptococcus equi subsp. zooepidemicus, Thoroughbred horse

\section{INTRODUCTION}

Equine herpes virus (EHV) is a common virus occurring in horse populations worldwide. The most common strains are EHV-1 and 4. EHV-1 can cause respiratory diseases, abortions and neurologic disease, while EHV-4 typically causes respiratory disease but can also cause abortions (Azmi and Field, 1993; Ostlund, 1993). EHV-1 has been identified as a cause of abortion outbreaks and, more recently, outbreaks of neurologic disease (Borchers et al, 2006). Respiratory disease caused by EHV is most common in young horses (weanlings and yearlings), while older horses are more likely to transmit the virus without showing symptoms (Reed and Toribio, 2004).

Streptococcus equi subsp. zooepidemicus (S. zooepidemicus) belongs to the $\beta$-hemolytic Group C streptococci, which can cause disease both in animals and humans. S. zooepidemicus may be found in the nasopharynx, tonsils, respiratory tract, and on the genital mucous membrane of healthy horses. It is an important cause of respiratory tract infections in foals and young horses, and associated with uterine infections in

*Corresponding author: Seong-Kyoon Choi, Tel. + 82-53-326-0013, Fax.+82-53-326-1066, E-mail. cskbest@hanmail.net mares (Anzai et al, 2002; Korman et al, 2004). Damage from Herpesvirus infection to the protective respiratory mucosal barrier predisposes affected horses to opportunistic bacterial infections. As a result, secondary bacterial rhinopharyngitis (primarily by $S$. zooepidemicus) commonly accompanies EHV infection and contributes to the overall severity of the illness. In the absence of appropriate antibiotic therapy, involvement of the lower airways with the consequent development of bacterial tracheobronchitis, bronchiolitis, or pneumonia can further complicate upper respiratory tract disease initiated by EHV-1 or EHV-4. Secondary invasion by clonally derived $S$. zooepidemicus strains with enhanced virulence can dramatically influence the severity and duration of the disease episode and may be associated with increased levels of morbidity and greater risks for mortality (Timoney, 2004).

\section{CASE AND DISCUSSION}

Horse with respiratory distress was died and requested to the Gyeongbuk Veterinary Service Laboratory. Gross 
necropsy revealed epistaxis, hyperemia on the lung, multiple hemorrhage in muscle, and liver was focally attached to the peritoneum with fibrin (Fig. 1).

Molecular identification of EHV and S. zooepidemicus isolates was performed by polymerase chain reaction (PCR). The glycoprotein B coding region of EHV and SodA gene, $S z P$ gene, $C N E$ virulence factor, and $16 \mathrm{~S}$ rRNA gene of $S$. zooepidemicus were then amplified with the oligonucleotide primers described by Younan et al.
(2005) and office international des epizooties Chapter 12. 1. (OIE, 2008), respectively. The sequences of the oligonucleotide primers are given in Table 1.

According to PCR, EHV-1 and 4 were detected in the lung and trachea of Thoroughbred horse. In bacterial culture from heart, kidney, liver, spleen, muscle, and blood, $S$. zooepidemicus was isolated from all cultured samples. The EHV-1 and 4 was confirmed by PCR with the amplicon sizes of $770 \mathrm{bp}$ and $580 \mathrm{bp}$, respectively.

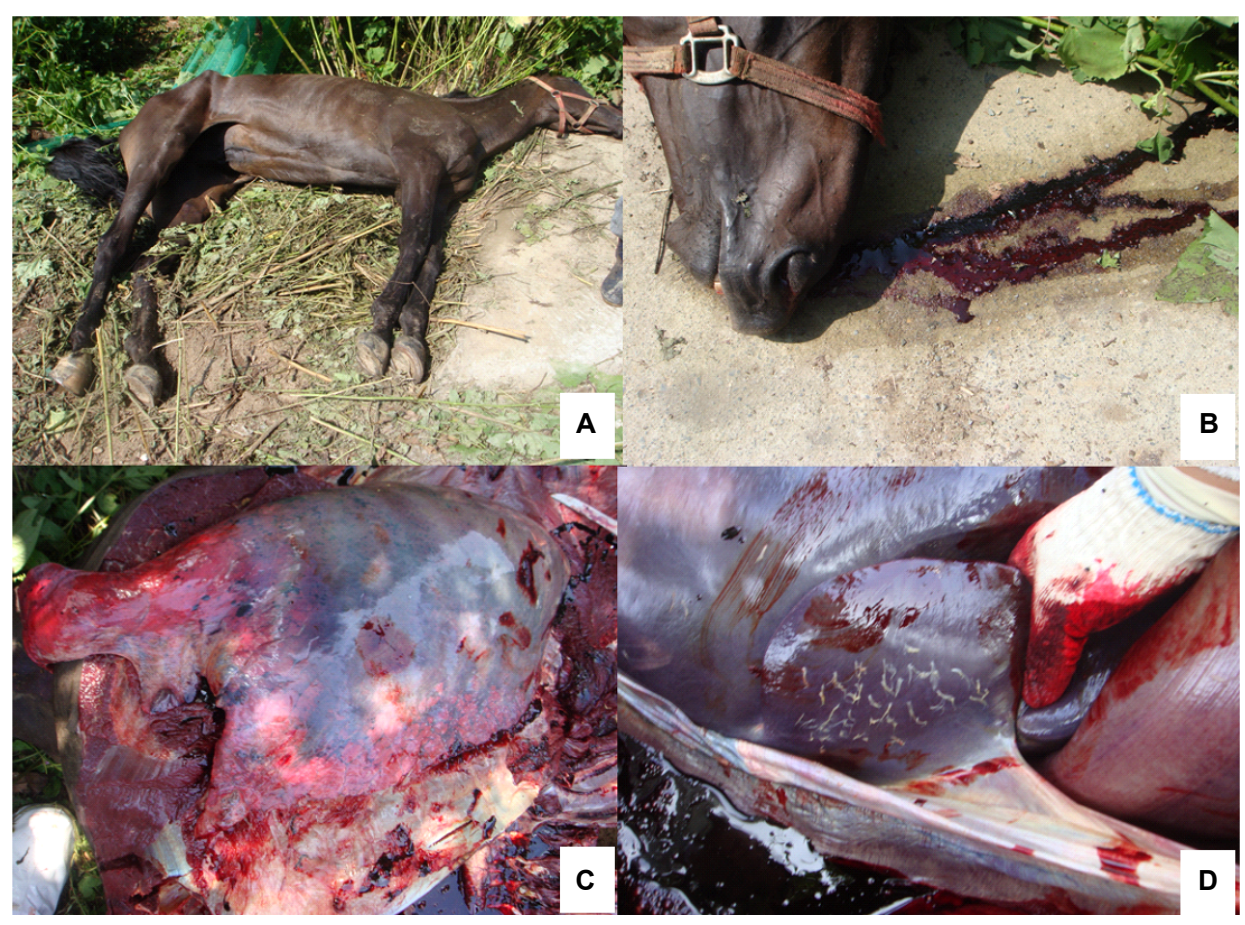

Fig. 1. Images from gross necropsy. (A) Emaciation; (B) Epistaxis; (C) Hyperemia and hemorrhage on the lung with lobular adhesion; (D) Liver attached to the peritoneum with fibrin.

Table 1. Oligonucleotide primers used in present study

\begin{tabular}{|c|c|c|c|}
\hline Target antigen & Primer & Sequence $\left(5^{\prime} \rightarrow 3^{\prime}\right)$ & Size of product (bp) \\
\hline \multirow[t]{10}{*}{ S. zooepidemicus } & \multirow[t]{2}{*}{ SodA } & CAGCATTCCTGCTGACATTCGTCAGG & \multirow[t]{2}{*}{235} \\
\hline & & CTGACCAGCCTTATTCACAACCAGC & \\
\hline & \multirow[t]{2}{*}{ SeeI } & GAAGGTCCGCCATTTTCAGGTACTTTG & \multirow[t]{2}{*}{520} \\
\hline & & GCATACTCTCTCTGTCACCATGTCCT & \\
\hline & \multirow[t]{2}{*}{$C N E$} & GCAACTAATCTTAGTGACAACAT & \multirow[t]{2}{*}{906} \\
\hline & & AAAGCTGGTATAGCGACTGCCA & \\
\hline & \multirow[t]{2}{*}{$I S R^{*}$} & TTGTACACACCGCCCGTCA & \multirow[t]{2}{*}{ Size polymorphism } \\
\hline & & GGTACCTTAGATGTTTCAGTT & \\
\hline & \multirow[t]{2}{*}{$S z P$} & ACAAAAGGGGAATAAAATGGC & \multirow[t]{2}{*}{ Size polymorphism } \\
\hline & & TTTACCACTGGGGTATAAGGCT & \\
\hline \multirow[t]{4}{*}{$\mathrm{EHV}^{* *}$} & \multirow[t]{2}{*}{ EHV-1 } & CTTTAGCGGTGATGTGGAA & \multirow[t]{2}{*}{770} \\
\hline & & AAGTAGCGCTTCTGATTGAG & \\
\hline & \multirow[t]{2}{*}{ EHV-4 } & TGTTTCCGCCACTCTTGAC & \multirow[t]{2}{*}{580} \\
\hline & & АCТGCСТCТСССАССТТАC & \\
\hline
\end{tabular}

*ISR: 16S rRNA gene, **EHV: Equine herpesvirus. 

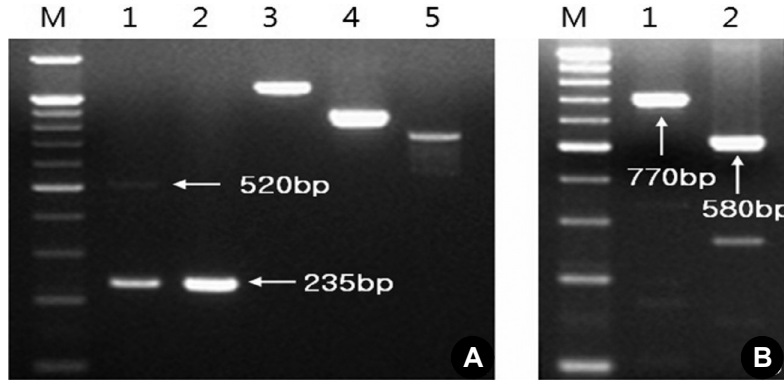

Fig. 2. PCR amplification for the identification of S. zooepidemicus and EHV type 1 and 4. Pannel A: Lane M, molecular size marker (100 bp DNA ladder, Elpis, Korea); Lane 1, S. equi ATCC 9528; Lane 2: S. zooepidemicus isolates; Lane 3, SzP encoding gene; Lane 4, CNE encoding gene; Lane 5, 16S rRNA gene(ISR). Pannel B: Lane M, molecular size marker (100 bp DNA ladder, Elpis, Korea); Lane 1, EHV-1; Lane 2, EHV-4.

S. zooepidemicus was identified by SodA gene PCR with one amplicon (235 bp), while $S$. equi has two (235 bp and $520 \mathrm{bp}$ ). S. zooepidemicus was positive for the $S z P$ gene with amplicon size of approximately 1,200 bp. Additionally, $C N E$, a 657 amino acid collagen-binding protein encoding gene of $S$. zooepidemicus was also detected. Typical amplicons of the $S z P, C N E$ and ISR genes are shown in Fig. 2.

On the bases of findings at autopsy such as hemorrhage of upper respiratory tract, pneumonia, and epistaxis, we diagnosed this case as equine viral rhinopneumonitis. We conducted exact diagnosis using PCR as well as bacteria test for septicemia, which was suspected to have been caused by secondary bacterial infection, based on the systemic organ hemorrhage and hyperemia. After final diagnosis using PCR, we could confirm that it was due to the coinfection of EHV-1, -4 and S. zooepidemicus, important causative agent of upper respiratory tract infection of the horse. Also, S. zooepidemicus isolated from the systemic organs including blood, spleen, kidney, liver, lung and muscle, showed positive reaction to both $S z P$ and $C N E$, the virulence factor of $S$. zooepidemicus. Therefore, in this case, the coinfection of EHV-1 and -4 caused equine viral rhinopneumonitis, and the immunodepression by viral infection resulted in the pathogenic $S$. zooepidemicus infection and septicemia, which led to the ultimately death.

Generally, relatively light symptoms accompany equine viral rhinopneumonitis caused by primary infection of
EHV-1 or -4 , but secondary infection on respiratory system can bring serious symptoms (Ostlund, 1993; Timoney, 2004). However, coinfections of these two rarely leads to death, and there has been no case report in Korea that involves simultaneous infection of EHV-1 and -4 , or that shows symptoms due to respiratory infection of $S$. zooepidemicus. Thus, the infectious disease diagnosed in this case seems to be very rare, and the coinfection of EHV-1, -4 and $S$. zooepidemicus is deemed fatal. In addition, we found that the management conditions for the horse were not so satisfactory since there has been no vaccination for critical diseases and proper treatment. In order to prevent the disease caused by those agents, appropriate vaccination and antibiotics treatment, if necessary, are required. Also, when considering that no horse has been newly imported to the farm and it is located in a relatively segregated area, this investigation points out that many strains of EHV and S. zooepidemicus could be isolated in horses, which correlates well with the fact that EHV and $S$. zooepidemicus are mucosal commensal organisms in the equine population, and the opportunistic pathogenic characters of thesemicroorganisms (Ostlund, 1993; Timoney, 2004).

\section{REFERENCES}

Anzai T, Timoney JE, Kuwamoto Y, Wada R, Oikawa M, Higuchi T. 2002. Polymerase chain reaction-restriction fragment length polymorphism analysis of the SzP gene of Streptococcus zooepidemicus isolated from the respiratory tract of horses. Am J Vet Res 63(9): 1298-1301.

Azmi M, Field HJ. 1993. Interactions between equine herpesvirus type 1 and equine herpesvirus type 4: T cell responses in a murine infection model. J Gen Virol 74(11): 23392345.

Borchers K, Thein R, Sterner-Kock A. 2006. Pathogenesis of equine herpesvirus- associated neurological disease: a revised explanation. Equine Vet J 38(3): 283-287.

Korman TM, Boers A, Gooding TM, Curtis N, Visvanathan K. 2004. Fatal case of toxic shock-like syndrome due to group $\mathrm{C}$ streptococcus associated with superantigen exotoxin. J Clin Microbiol 42(6): 2866-2869.

Office International Des Epizooties (OIE). 2008. Manual of diagnostic tests and vaccines for terrestrial animals, Vol. II. 6th Edition. World Organization for Animal Health, Paris, France. Capter 12. 1. 
Ostlund EN. 1993. The equine herpesviruses. Vet Clin North Am Equine Pract 9(2): 283-294.

Patel JR, Heldens J. 2005. Equine herpesviruses 1 (EHV-1) and 4 (EHV-4) epidemiology, disease and immunoprophylaxis: a brief review. Vet J 170(1): 14-23.

Reed SM, Toribio RE. 2004. Equine herpesvirus 1 and 4. Vet Clin North Am Equine Pract 20(3): 631-642.

Sutton GA, Viel L, Carman PS, Boag BL. 1998. Pathogenesis and clinical signs of equine herpesvirus-1 in experimentally infected ponies in vivo. Can J Vet Res
62(1): 49-55.

Timoney JF. 2004. The pathogenic equine streptococci. Vet Res 35(4): 397-409.

Younan M, Estoepangestie AT, Cengiz M, Alber J, El-Sayed A, Lämmler C. 2005. Identification and molecular characterization of Streptococcus equi subsp. zooepidemicus isolated from camels (Camelus dromedarius) and camel milk in Kenya and Somalia. J Vet Med B Infect Dis Vet Public Health 52(3): 142-146. 